\title{
Improved Dysphagia After Decannulation of Tracheostomy in Patients With Brain Injuries
}

\author{
Yong Kyun Kim, MD, PhD, Jung-Hwa Choi, MD, Jeong-Gyu Yoon, MD, \\ Jang-Won Lee, MD, Sung Silk Choi, MD
}

Department of Physical Medicine and Rehabilitation, Myongji Hospital, Seonam University College of Medicine, Goyang, Korea

\begin{abstract}
Objective To investigate improved dysphagia after the decannulation of a tracheostomy in patients with brain injuries.

Methods The subjects of this study are patients with brain injuries who were admitted to the Department of Rehabilitation Medicine in Myongji Hospital and who underwent a decannulation between 2012 and 2014. A video fluoroscopic swallowing study (VFSS) was performed in order to investigate whether the patients' dysphagia had improved. We measured the following 5 parameters: laryngeal elevation, pharyngeal transit time, postswallow pharyngeal remnant, upper esophageal width, and semisolid aspiration. We analyzed the patients' results from VFSS performed one month before and one month after decannulation. All VFSS images were recorded using a camcorder running at 30 frames per second. An AutoCAD 2D screen was used to measure laryngeal elevation, post-swallow pharyngeal remnant, and upper esophageal width.

Results In this study, a number of dysphagia symptoms improved after decannulation. Laryngeal elevation, pharyngeal transit time, and semisolid aspiration showed no statistically significant differences ( $>>0.05)$, however after decannulation, the post-swallow pharyngeal remnant (pre $37.41 \% \pm 24.80 \%$, post $21.02 \% \pm 11.75 \%$; $<<0.001$ ) and upper esophageal width (pre $3.57 \pm 1.93 \mathrm{~mm}$, post $4.53 \pm 2.05 \mathrm{~mm} ; \mathrm{p}<0.001$ ) showed statistically significant differences.

Conclusion When decannulation is performed on patients with brain injuries who do not require a ventilator and who are able to independently excrete sputum, improved esophageal dysphagia can be expected.
\end{abstract}

Keywords Fluoroscopy, Tracheostomy, Dysphagia, Brain injuries, Upper esophageal sphincter

Received November 13, 2014; Accepted May 15, 2015

Corresponding author: Sung Sik Choi

Department of Physical Medicine and Rehabilitation, Myongji Hospital, Seonam University College of Medicine, 55 Hwasu-ro 14beon-gil, Deokyang-gu, Goyang 10475, Korea

Tel: +82-31-810-6450, Fax: +82-31-810-5259, E-mail: saintc@naver.com

(c) This is an open-access article distributed under the terms of the Creative Commons Attribution Non-Commercial License (http://creativecommons. org/licenses/by-nc/4.0) which permits unrestricted noncommercial use, distribution, and reproduction in any medium, provided the original work is properly cited.

Copyright $\odot 2015$ by Korean Academy of Rehabilitation Medicine

\section{INTRODUCTION}

Tracheostomy is a common surgical treatment for artificial ventilation, sputum excretion, and airway maintenance in critically ill patients [1]. The procedure is performed in $10 \%$ of patients with mechanical ventilation for airway maintenance and continuous respiration maintenance. Patients who require long-term mechanical ventilation or have difficulty excreting sputum on their own continuously maintain a tracheostomy tube to aid airway 
maintenance and sputum excretion [2-4]. Tracheostomy is also performed in patients with ischemic or hemorrhagic stroke to resolve their prolonged inability to sufficiently breathe and/or protect their airways [5]. However, if the tracheostomy tube is maintained for a lengthy duration, various complications may occur such as tracheal stenosis, bleeding, fistula, infection, aspiration, airway ciliary damage and dysfunction, increase in excretion caused by airway stimulus, and dysphagia, among others [6-8]. In particular, the prevalence of dysphagia after a tracheostomy is not exactly known and varies widely in the literature (between $50 \%$ and $83 \%$ ) [9].

Decannulation is considered only if the patient does not use a ventilator and can manage secretion without assistance [10]. In particular, because frequent aspiration is considered to be a relative contraindication for decannulation, the risk of aspiration should be checked in consideration of the procedure [11]. In addition, airway patency must be examined by laryngoscopy for tracheal stenosis, subglottic stenosis, glottic stenosis, and tracheal granuloma among other potential difficulties [12]. Furthermore, when corking, the oxygen saturation of the room air must be maintained at $92 \%$ or above, and the peak cough flow must be at least $160 \mathrm{~L} / \mathrm{min}$ [11]. If these conditions are met and tolerance for corking does exist, decannulation can be safely performed [10].

If patients show improvement in dysphagia after decannulation, decannulation should be considered, regardless of the low risk of aspiration. However, the influence of decannulation on dysphagia is still in question. There- fore, this study seeks to determine if dysphagia improves following decannulation in brain injury patients with tracheostomy.

\section{MATERIALS AND METHODS}

The Myongji Hospital ethics committee approved this study. The Institutional Review Board number is 14-047.

\section{Methods}

\section{Subjects}

This study was conducted with 17 brain injury patients who had been admitted to the rehabilitation medicine department of Myongji Hospital between May 1, 2012, and February 28, 2014.

The inclusion criteria were as follows: patients with brain injuries from cerebral hemorrhage and/or infarction, traumatic brain injury, or brain hypoxia, patients with brain injury who underwent decannulation, and patients who had VFSS within 1 month prior to the decannulation and again within 1 month after the procedure.

The exclusion criteria were as follows: patients with structural abnormality in the oropharynx and patients with structural abnormality in the cervical spine (for example, bone abnormalities, such as osteophyte, etc.).

\section{Measures}

VFSS was performed while patients swallowed a semisolid solution (porridge, step 1 diet to treat dysphagia), and the following 5 factors were measured: laryngeal ele-
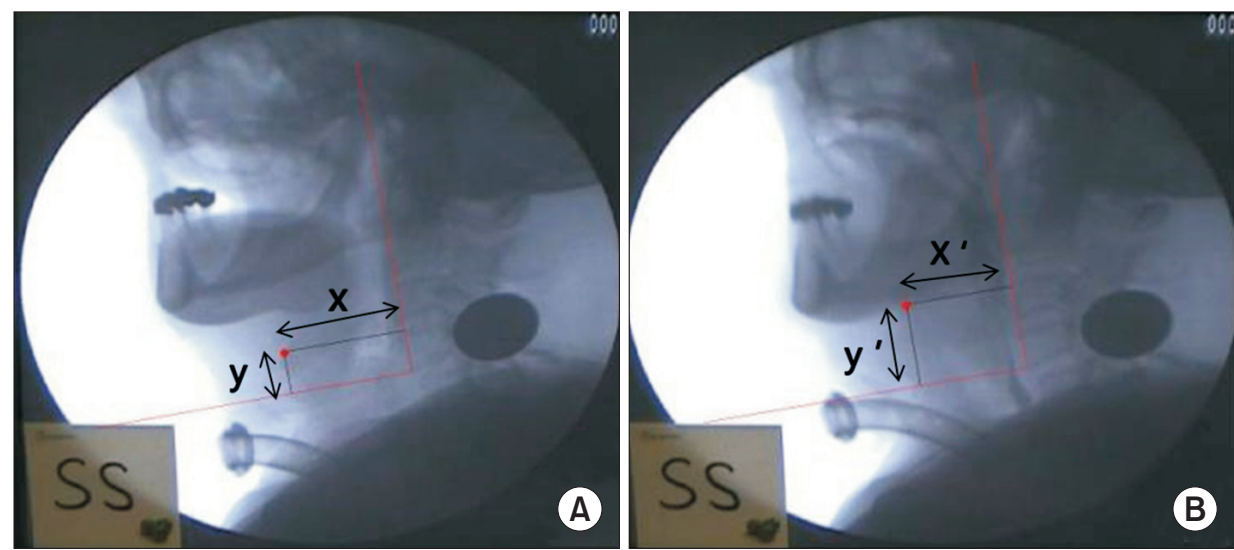

Fig. 1. Laryngeal elevation. The zero point is defined as the anterior-inferior margin of the fourth cervical vertebral body, with the $y$-axis as the straight line that connects the zero point with the anterior-inferior margin of the second cervical vertebral body and the $\mathrm{x}$-axis as the line perpendicular to the $\mathrm{y}$-axis. The hyoid bone shown in its position in the resting state (A) and at the most highly raised position during swallowing (B). 
vation, pharyngeal transit time, post-swallow pharyngeal remnant, upper esophageal width, and semisolid aspiration. For laryngeal elevation, a straight line was drawn from the anterior-inferior margin of the 4th vertebral body to the anterior-inferior margin of the 2 nd vertebral body as the $\mathrm{y}$-axis, and the $\mathrm{x}$-axis was set perpendicular to the $y$-axis to measure the change in location of the hyoid bone before and after swallowing ( $\mathrm{mm}$ ) [13] (Fig. 1A, B). The pharyngeal transit time was measured as the time (s) that it took for food to pass from the posterior nasal spine to the pharyngoesophageal sphincter [14]. The post-swallow pharyngeal remnant was represented as the initial remnant and the remnant after swallowing the food once (Fig. 2A, B). The upper esophageal width was measured as the anteroposterior diameter $(\mathrm{mm})$ of the narrowest region in C3-6 [15] (Fig. 3A, B). Semisolid aspiration was examined with VFSS and evaluated with the penetration-aspiration scale (PAS).

\section{Procedure}

VFSS was performed with a 3-mL bolus of semisolid.
This study used the semisolid to calculate the amount of remnant. The VFSS test was performed in a chintucked position for all patients. Patients with cognitive disorders and those who could not actively control their head positions were seated in a wheelchair, with assistants holding their heads to maintain a chin-tucked position. Every VFSS was recorded with a camcorder at a rate of 30 frames per second. All images were saved on a personal computer and analyzed with a multimedia player (Gretech, Seoul, Korea). Additionally, laryngeal elevation, post-swallow pharyngeal remnant, and upper esophageal width were measured with 2D screen AutoCAD (Autodesk, San Francisco, CA, USA), and the guideline for length was a 100 Won Korean coin attached to the neck (23 $\mathrm{mm}$ in diameter).

\section{Statistical analysis}

The 5 factors measured in this study were analyzed with Wilcoxon signed-rank tests to compare before and after decannulation results. All statistical analyses were performed with SPSS ver. 18.0. (SPSS Inc., Chicago, IL, USA).
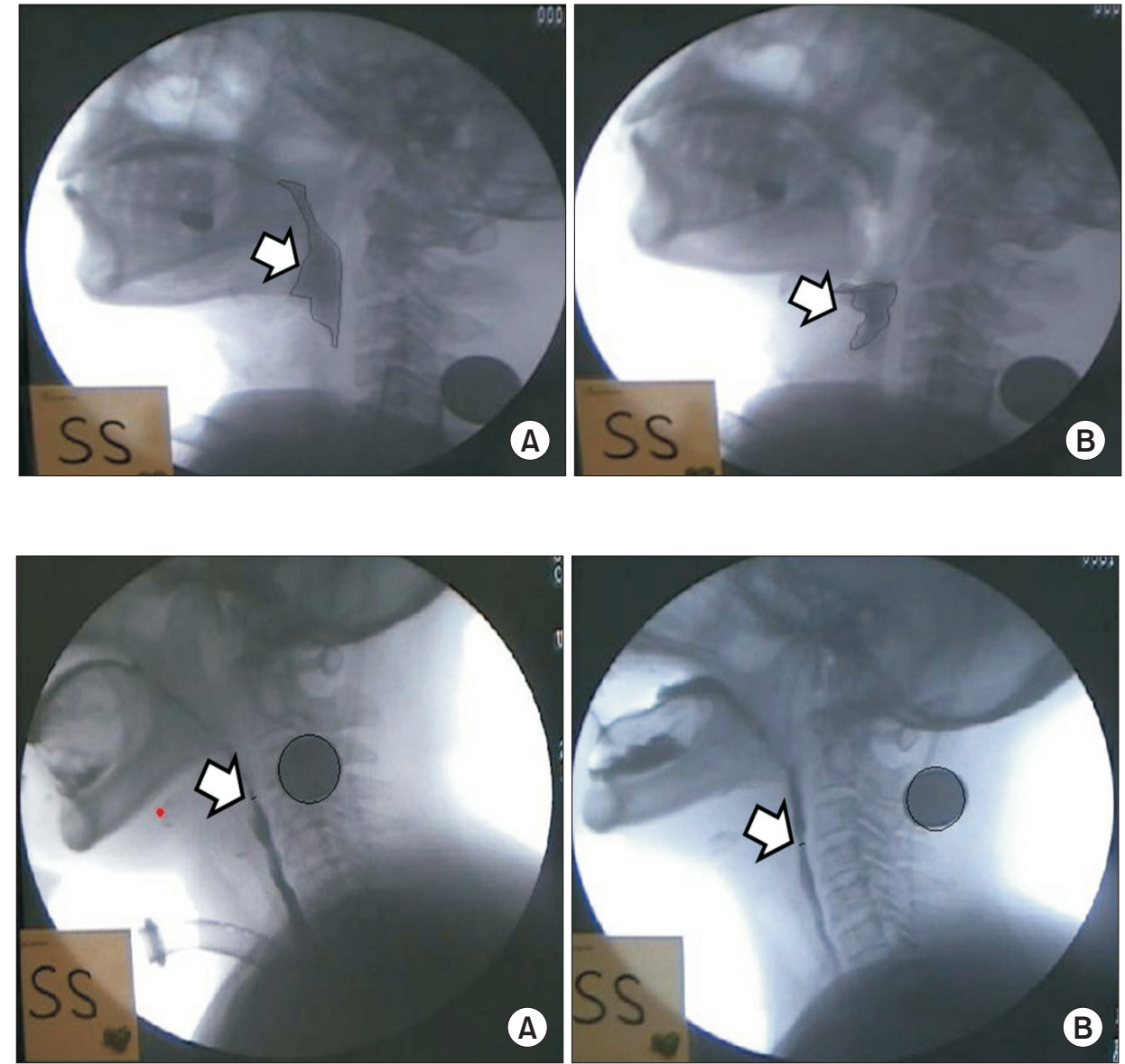

Fig. 3. Upper esophageal width. Corresponds to the anteroposterior diameter $(\mathrm{mm})$ of the narrowest region of C3-6 during maximum opening using the AutoCAD 2D screen. (A) Resting state and (B) swallowing state. 
The $p$-values less than 0.05 were considered significant.

\section{RESULTS}

The average period from onset to decannulation was $423.35 \pm 250.22$ days and the average age of the patient group was $58.88 \pm 14.05$ years. The average interval from pre- to post-decannulation VFSS was $26.65 \pm 12.94$ days (Table 1).

There were no statistically significant differences in laryngeal elevation ( $\mathrm{x}$-axis: pre $2.60 \pm 1.47 \mathrm{~mm}$ and post
$3.17 \pm 2.10 \mathrm{~mm}, \mathrm{p}=0.356$; $\mathrm{y}$-axis: pre $10.46 \pm 4.31 \mathrm{~mm}$ and post $12.45 \pm 5.46 \mathrm{~mm}, \mathrm{p}=0.287$ ), pharyngeal transit time (pre $8.99 \pm 11.90$ seconds and post $8.83 \pm 10.21 \mathrm{sec}$ onds, $\mathrm{p}=0.287$ ), or semisolid aspiration (PAS score: pre $4.24 \pm 3.23$ and post $3.88 \pm 3.24, \mathrm{p}=0.180$ ). However, there were statistically significant differences in post-swallow pharyngeal remnant (pre $37.41 \% \pm 24.80 \%$ and post $21.02 \% \pm 11.75 \%, \mathrm{p}<0.001)$ and upper esophageal width (pre $3.57 \pm 1.93 \mathrm{~mm}$ and post $4.53 \pm 2.05 \mathrm{~mm}, \mathrm{p}<0.001$ ) (Table 2).

Table 1. Participant characteristics

\begin{tabular}{ccclccc}
\hline $\begin{array}{c}\text { Patient } \\
\text { no. }\end{array}$ & Sex & $\begin{array}{c}\text { Age } \\
\text { (yr) }\end{array}$ & \multicolumn{1}{c}{ Diagnosis } & $\begin{array}{c}\text { Time from brain } \\
\text { injury to VFSS } \\
\text { (day) }\end{array}$ & $\begin{array}{c}\text { Duration of } \\
\text { tracheostomy } \\
\text { (day) }\end{array}$ & $\begin{array}{c}\text { Time between VFSS } \\
\text { before/after } \\
\text { decannulation (day) }\end{array}$ \\
\hline 1 & M & 63 & ICH & 397 & 403 & 16 \\
\hline 2 & M & 54 & ICH & 65 & 76 & 30 \\
\hline 3 & F & 47 & HBI & 208 & 213 & 30 \\
\hline 4 & M & 53 & ICH & 386 & 409 & 50 \\
\hline 5 & F & 67 & ICH & 405 & 651 & 33 \\
\hline 6 & M & 60 & TBI & 240 & 419 & 14 \\
\hline 7 & M & 66 & HBI & 330 & 237 & 7 \\
\hline 8 & M & 66 & TBI & 295 & 326 & 28 \\
\hline 9 & M & 66 & ICH & 693 & 291 & 8 \\
10 & F & 69 & ICH & 1,120 & 690 & 30 \\
11 & M & 40 & ICH & 311 & 1,085 & 21 \\
12 & M & 59 & ICH & 326 & 307 & 49 \\
\hline 13 & M & 60 & IFC & 821 & 345 & 21 \\
\hline 14 & F & 22 & HBI & 310 & 817 & 16 \\
\hline 15 & F & 85 & Cerebellar infarction & 301 & 309 & 42 \\
\hline 16 & M & 52 & HBI & 301 & 300 & 21 \\
\hline 17 & M & 72 & MCA infarction & 319 & \\
\hline
\end{tabular}

ICH, intra-cerebral hemorrhage; HBI, hypoxic brain injury; TBI, traumatic brain injury; MCA, middle cerebral artery.

Table 2. Changes in laryngeal elevation, pharyngeal transit time, post-swallow pharyngeal remnant, upper esophageal width, and semisolid aspiration after decannulation

\begin{tabular}{lccc}
\hline \multicolumn{1}{c}{ Variable } & Before decannulation & After decannulation & p-value \\
\hline Laryngeal elevation x-axis (mm) & $2.60 \pm 1.47$ & $3.17 \pm 2.10$ & 0.256 \\
\hline Laryngeal elevation y-axis (mm) & $10.46 \pm 4.31$ & $12.45 \pm 5.46$ & 0.287 \\
pharyngeal transit time (s) & $8.99 \pm 11.90$ & $8.83 \pm 10.21$ & 0.287 \\
Semisolid aspiration (PAS score) & $4.24 \pm 3.23$ & $3.88+3.24$ & 0.180 \\
Post-swallow pharyngeal remnant (\%) & $37.41 \pm 24.80$ & $21.02 \pm 11.75$ & $<0.001$ \\
\hline Upper esophageal width (mm) & $3.57 \pm 1.93$ & $4.53 \pm 2.05$ & $<0.001$ \\
\hline
\end{tabular}

Values are presented as mean \pm standard deviation.

PAS, penetration-aspiration scale. 


\section{DISCUSSION}

In our study, we found a statistically significant difference in post-swallow pharyngeal remnant and upper esophageal width after decannulation in brain injury patients who underwent tracheostomy.

In a previous study performed by Kang et al. [16] of 11 stroke patients, including one spinal cord injury patient and one panperitonitis patient, the researchers found no improvement in laryngeal elevation, pharyngeal transit time, or aspiration after decannulation. The present study limited its patient group to only those with brain injuries, and it included two additional measures, postswallow pharyngeal remnant and upper esophageal width in order to examine dysphagia improvement. With regard to improvement in dysphagia after decannulation, the present study did not find statistically significant differences in laryngeal elevation, pharyngeal transit time, or semisolid aspiration, just as in the study by Kang et al. [16]. The present study did, however, find statistically significant differences in post-swallow pharyngeal remnant and upper esophageal width. From a theoretical point of view, the tracheostomy cannula may impair the normal swallowing process via the following mechanisms: reduced laryngeal elevation (mainly if the cuff is inflated), reduced laryngeal sensitivity and cough reflex, lack of subglottic pressure, and poorer coordination between swallowing and breathing, especially in patients suffering from a neuromuscular disease or chronic obstructive pulmonary disease [9]. Therefore, decannulation should theoretically result in the recovery of the factors mentioned above.

The factor that is considered to be the most important in improving dysphagia after decannulation is deglutitive subglottic pressure. Subglottic air pressure is known to play an important role in swallowing motor control [17]. Subglottic air pressure stimulates the trachea's subglottic pressure receptor, thereby interfering with the swallowing function [18]. This pressure can occur with normal swallowing and is decreased in tracheostomy patients [19]. Low subglottic air pressure can worsen the swallowing-breathing interaction in tracheostomy patients and increase the risk of penetration and aspiration because of a damaged airway protection mechanism [9]. The literature mentions that a unidirectional tracheostomy speaking valve can block expiratory airflow to the trache- ostomy tube. The valve can therefore reinforce expiratory airflow to the upper airway and larynx, maintaining positive subglottic air pressure. This unidirectional valve can aid in safe swallowing by reducing laryngeal penetration and aspiration [20]. Similarly, there are a number of other ways to induce tracheal occlusion by creating deglutitive subglottic pressure-the Passy-Muir speaking valve, digital occlusion, capping, and decannulation [19]. Furthermore, keeping the subglottic system closed so that the pressure can be increased can also strengthen swallowing [21]. We applied dressings to the decannulation site during the decannulation procedure (Fig. 4). The dressings can prevent air leakage through the decannulation site, which would block the expiratory inflow, and as a result, positive subglottic air pressure is restored. Therefore, dressing the decannulation site after the procedure may improve dysphagia. If the site is sealed off or a stomaplasty is performed, the prognosis is considered to be better. To further investigate this finding, a study that examines the differences between sealed-off group and non-sealed-off groups may be required.

Previously, it was understood that swallowing dysfunction occurs because of the 'anchoring' of the larynx to the anterior neck after a tracheostomy [16]. Therefore, it was a common understanding that decannulation reduced this anchoring effect by increasing laryngeal elevation.

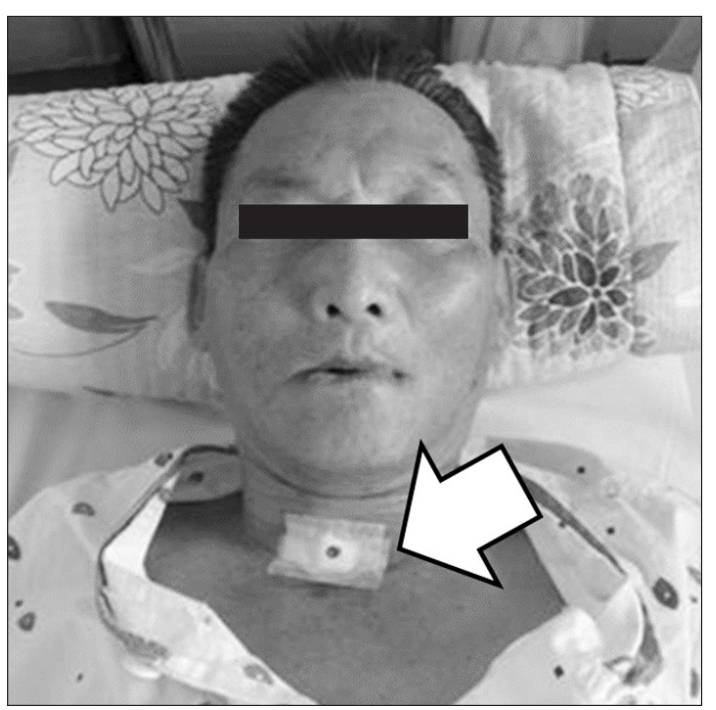

Fig. 4. Method of sealing the offsite dressing. The comma (arrow) indicates the seal off site. Pressing the area of dressing site prevents air leakage into the seal off site in case of a cough. 
However, recent studies have shown no differences in laryngeal elevation before and after decannulation $[16,22]$, which agrees with the results of the present study.

This study also found no significant differences in the pharyngeal transit time after decannulation. This result agrees with the work of Kang et al. [16], who showed that decannulation itself has no kinematic effect on the swallowing mechanism.

Some studies argue that aspiration increases after a tracheostomy $[16,23]$. However, in a number of other studies, aspiration did not improve after decannulation $[24,25]$. It appears that decannulation itself cannot fully restore the protective mechanisms that were damaged by the tracheostomy. Among the mechanisms that prevent aspiration, laryngeal elevation did not significantly improve after decannulation, as confirmed by other studies [10]. In the present study, decannulation itself did not significantly improve laryngeal elevation, and for this reason, it appears that decannulation did not improve aspiration.

The reasons for the existence of the post-swallow pharyngeal remnant after swallowing are either that the tongue base retraction is weak, creating an insufficient pharyngeal propulsive force, or that the upper esophageal sphincter (UES) opening is insufficient [10]. Therefore, in this study, improved post-swallow pharyngeal remnant after decannulation would only result after improved tongue base retraction base. However, this procedure requires measurement with the Iowa Oral Performance Instrument (IOPI), and thus, additional studies are required. The IOPI can measure the strength of the tongue and lip [26]. In contrast, with regard to the possibility of reducing the post-swallow pharyngeal remnant by improving the UES opening, we found no statistically significant correlation between changes in UES width and changes in the post-swallow pharyngeal remnant $(p=0.510)$ in this study. This could be because of the study's small sample, and thus, a larger sample size will be required to further confirm this result. Meanwhile, if a patient has a cuffed-type tracheostomy, compression in the esophagus may be because of the cuff within the trachea [23]. In the present study, all patients had uncuffed type tracheostomies. Therefore, the precise reason for the improvement in the post-swallow pharyngeal remnant after decannulation has not yet been elucidated, and further study is required. Improving the UES opening did appear to have some influence, and this could be because of improved deglutitive subglottic pressure, which would further improving the tongue's retraction.

The UES is kept shut by the tonic contraction of the cricopharyngeus muscle during swallowing, but between each swallow, it opens briefly [27]. Three major factors combine to cause the UES to open: relaxing the cricopharyngeus muscle opens the sphincter, constricting the suprahyoid and thyrohyoid muscles moves the larynx to the front, opening the sphincter, and the hydrostatic pressure created as the bolus comes down pushes the UES to the exterior, opening it [28]. In this study, after decannulation, improved upper esophageal width was observed. Thus, decannulation can be considered effective in improving the UES opening. The increased upper esophageal width may be because the cricopharyngeus muscle relaxes after decannulation. However, to fully elucidate the mechanism, the pressure of the cricopharyngeus muscle must be measured. This measurement requires high-resolution impedance manometry in addition to VFSS [29]. The increased upper esophageal width may also be a result of the stronger contraction of the suprahyoid and thyrohyoid muscles after decannulation. However, as was indicated in various studies, laryngeal elevation does not show a significant difference after decannulation, which makes it possible to rule out this cause. Finally, an increase in the hydrostatic pressure while the patient swallows a bolus after decannulation may lead to increased upper esophageal width, although because the same amount of bolus $(3 \mathrm{~mL})$ was applied each time, it appears that hydrostatic pressure does not cause the increase. Therefore, measurement by high-resolution impedance manometry before and after decannulation is required to establish the precise reason for the improved UES width after decannulation.

There are some limitations to this study. The first is that the patient group consisted of patients who received rehabilitation in the Myongji Hospital rehabilitation medicine department and who continuously received conventional dysphagia therapies. The average interval between VFSS before and after decannulation was $26.6 \pm 12.9$ days, and even during this period, the group was continuously treated with dysphagia therapy. However, two cases showed improvement in the pharyngeal remnant and UES even though the intervals between the VFSSs before and after decannulation were short. The case with a sev- 
en-day interval showed improvement in both post-swallow pharyngeal remnant (pre $15.25 \%$, post $0 \%$ ) and UES width (pre $6.94 \mathrm{~mm}$, post $7.34 \mathrm{~mm}$ ). In addition, another case with an eight-day interval showed improvement in both post-swallow pharyngeal remnant (pre $17.70 \%$, post $10.78 \%$ ) and UES width (pre $2.81 \mathrm{~mm}$, post $3.68 \mathrm{~mm}$ ).

Second, a part that spontaneously recovered over time exists in brain injury patients with dysphagia. According to Drulia and Ludlow [17], patients who experienced stroke within 6 months after onset were defined as the subacute group. Regarding subacute stroke, it has been said that the proportion of spontaneous recovery was greater than that of recovery under treatment [30], and in addition, most stroke patients developed functional recovery from dysphagia in several days or weeks [18]. In some studies, dysphagia improved even a few months or years after stroke, but the improvement was reportedly slow $[31,32]$. In light of this information, the period from the onset of brain injury until decannulation in the patient group in this study was long, with an average of $436.0 \pm 251.2$ days, and in all cases except one, more than 6 months had passed since the onset. Therefore, it seems that the role of spontaneous recovery was not large in the present study.

The third limitation is that the tracheostomy tubes were maintained for a long duration in the patient group, an average of $423.35 \pm 250.22$ days. Because of this, various forms of adaptation during the long period after decannulation are possible [1].

Fourth, this study hypothesized that the cause of increased upper esophageal width was improved cricopharyngeus muscle relaxation, but to acquire a more accurate result, measuring the cricopharyngeus muscle's pressure using high-resolution impedance manometry will be required.

Finally, the small sample size is a limitation, so additional studies with larger sample sizes will be required to confirm our findings.

In conclusion, if decannulation is performed on a brain injury patient with dysphagia, one may expect improvement in the post-swallow pharyngeal remnant and upper esophageal width. Therefore, when decannulation is performed on such patients who do not require a ventilator and who are able to excrete sputum physiologically, improvement of esophageal dysphagia can be expected.

\section{CONFLICT OF INTEREST}

No potential conflict of interest relevant to this article was reported.

\section{REFERENCES}

1. Stelfox HT, Crimi C, Berra L, Noto A, Schmidt U, Bigatello LM, et al. Determinants of tracheostomy decannulation: an international survey. Crit Care 2008;12:R26.

2. Frutos-Vivar F, Esteban A, Apezteguia C, Anzueto A, Nightingale $\mathrm{P}$, Gonzalez M, et al. Outcome of mechanically ventilated patients who require a tracheostomy. Crit Care Med 2005;33:290-8.

3. Kollef MH, Ahrens TS, Shannon W. Clinical predictors and outcomes for patients requiring tracheostomy in the intensive care unit. Crit Care Med 1999;27:1714-20.

4. Fischler L, Erhart S, Kleger GR, Frutiger A. Prevalence of tracheostomy in ICU patients. A nation-wide survey in Switzerland. Intensive Care Med 2000;26:1428-33.

5. Bosel J. Tracheostomy in stroke patients. Curr Treat Options Neurol 2014;16:274.

6. Goldenberg D, Ari EG, Golz A, Danino J, Netzer A, Joachims HZ. Tracheotomy complications: a retrospective study of 1130 cases. Otolaryngol Head Neck Surg 2000;123:495-500.

7. Epstein SK. Late complications of tracheostomy. Respir Care 2005;50:542-9.

8. Norwood S, Vallina VL, Short K, Saigusa M, Fernandez LG, McLarty JW. Incidence of tracheal stenosis and other late complications after percutaneous tracheostomy. Ann Surg 2000;232:233-41.

9. Ceriana P, Carlucci A, Schreiber A, Fracchia C, Cazzani C, Dichiarante $\mathrm{M}$, et al. Changes of swallowing function after tracheostomy: a videofluoroscopy study. Minerva Anestesiol 2015;81:389-97.

10. Braddom RL. Physical medicine and rehabilitation. 4th ed. Philadelphia: Saunders; 2011. p. 579-97.

11. Garuti G, Reverberi C, Briganti A, Massobrio M, Lombardi F, Lusuardi M. Swallowing disorders in tracheostomised patients: a multidisciplinary/multiprofessional approach in decannulation protocols. Multidiscip Respir Med 2014;9:36.

12. Wise SP, Murray EA, Gerfen CR. The frontal cortexbasal ganglia system in primates. Crit Rev Neurobiol 1996;10:317-56. 
13. Nam HS, Beom J, Oh BM, Han TR. Kinematic effects of hyolaryngeal electrical stimulation therapy on hyoid excursion and laryngeal elevation. Dysphagia 2013;28:548-56.

14. Kim JC, Kim JS, Jung JH, Kim YK. The effect of balloon dilatation through video-fluoroscopic swallowing study (VFSS) in stroke patients with cricopharyngeal dysfunction. J Korean Acad Rehabil Med 2011;35:23-6.

15. Wada S, Tohara H, Iida T, Inoue M, Sato M, Ueda K. Jaw-opening exercise for insufficient opening of upper esophageal sphincter. Arch Phys Med Rehabil 2012;93:1995-9.

16. Kang JY, Choi KH, Yun GJ, Kim MY, Ryu JS. Does removal of tracheostomy affect dysphagia? A kinematic analysis. Dysphagia 2012;27:498-503.

17. Drulia TC, Ludlow CL. Relative efficacy of swallowing versus non-swallowing tasks in dysphagia rehabilitation: current evidence and future directions. Curr Phys Med Rehabil Rep 2013;1:242-56.

18. Gross RD, Mahlmann J, Grayhack JP. Physiologic effects of open and closed tracheostomy tubes on the pharyngeal swallow. Ann Otol Rhinol Laryngol 2003;112:143-52.

19. Gross RD, Steinhauer KM, Zajac DJ, Weissler MC. Direct measurement of subglottic air pressure while swallowing. Laryngoscope 2006;116:753-61.

20. Gross RD, Mahlmann J, Grayhack JP. Physiologic effects of open and closed tracheostomy tubes on the pharyngeal swallow. Ann Otol Rhinol Laryngol 2003;112:143-52.

21. Eibling DE, Gross RD. Subglottic air pressure: a key component of swallowing efficiency. Ann Otol Rhinol Laryngol 1996;105:253-8.

22. Terk AR, Leder SB, Burrell MI. Hyoid bone and laryngeal movement dependent upon presence of a trache- otomy tube. Dysphagia 2007;22:89-93.

23. Feldman SA, Deal CW, Urquhart W. Disturbance of swallowing after tracheostomy. Lancet 1966;1:954-5.

24. Donzelli J, Brady S, Wesling M, Theisen M. Effects of the removal of the tracheotomy tube on swallowing during the fiberoptic endoscopic exam of the swallow (FEES). Dysphagia 2005;20:283-9.

25. Leder SB, Ross DA. Confirmation of no causal relationship between tracheotomy and aspiration status: a direct replication study. Dysphagia 2010;25:35-9.

26. Adams V, Mathisen B, Baines S, Lazarus C, Callister R. Reliability of measurements of tongue and hand strength and endurance using the Iowa Oral Performance Instrument with elderly adults. Disabil Rehabil 2015;37:389-95.

27. Ertekin C, Aydogdu I. Electromyography of human cricopharyngeal muscle of the upper esophageal sphincter. Muscle Nerve 2002;26:729-39.

28. Cook IJ, Dodds WJ, Dantas RO, Massey B, Kern MK, Lang IM, et al. Opening mechanisms of the human upper esophageal sphincter. Am J Physiol 1989;257(5 Pt 1):G748-59.

29. Kuhn MA, Belafsky PC. Management of cricopharyngeus muscle dysfunction. Otolaryngol Clin North Am 2013;46:1087-99.

30. Rehman HU, Knox J. There is a need for a regular review of swallowing ability in patients after PEG insertion to identify patients with delayed recovery of swallowing. Dysphagia 2000;15:48.

31. Hull MA, Rawlings J, Murray FE, Field J, McIntyre AS, Mahida YR, et al. Audit of outcome of long-term enteral nutrition by percutaneous endoscopic gastrostomy. Lancet 1993;341:869-72.

32. Singh S, Hamdy S. Dysphagia in stroke patients. Postgrad Med J 2006;82:383-91. 